\title{
Variable Switch Regenerative Braking Technique for PM BLDC Motor Driven Electric Two-wheeler
}

\author{
Manish Kumar Dubey ${ }^{1, *}$ and Phaneendra Babu Bobba ${ }^{2}$ \\ ${ }^{1}$ Research Centre Imarat, Kalam Missile Complex, DRDO, Hyderabad \\ ${ }^{2}$ EEE Department, GRIET, Hyderabad, India
}

\begin{abstract}
In this paper PM BLDC motor driven electric two-wheeler is proposed. The operation of PMBLDC motor in all four quadrants along with various regenerative braking control strategies are simulated MATLAB/ SIMULINK. Three braking methods are proposed for electric vehicle application. Factors like recovered energy, braking time, maximum braking current are compared. Depending on the simulation results switching between different braking methods is proposed for efficient and reliable braking operation. Moreover, by using variable switch braking technique one can extract more braking energy.
\end{abstract}

\section{Introduction}

Environmental pollution, rapid depletion and increase in the cost of fossil fuels are the significant factors which are stimulating the use of Electric Vehicles for the transportation purpose. These vehicles would also enable meeting the demands for electrical power due to the increasing use of electronic features to improve vehicle performance, fuel economy, emissions, passenger comfort, and safety. In electric vehicles the challenges are long range, efficient power electronics circuits and their control and electrical machines.

In an internal combustion engine powered vehicle, a multi-gear transmission is required to shape the speed torque profile of the engine to obtain an approximate constant power profile. In modem ICE vehicles are usually equipped with complex automatic transmission, which consists of multi-gear box. When vehicles are operated with more start and stop driving pattern, such as driving in cities, the average fuel consumption efficiency of the torque converter is very low (less than 60\%). Therefore, necessity of multi-gear transmission is unfavourable.

The desirable characteristics of an electric motor drive for traction application in an electric and hybrid electric vehicle are high starting torque at low speed region for fast acceleration, hill climbing and obstacle negotiation, and low torque at high speed for normal driving. Permanent Magnet Brushless DC motor (PM BLDC) is very efficient and compact motor, with good reliability and simple control. These motors find application mainly in two-wheeler and three-wheeler electric vehicles.

The bottleneck of the development of EV technologies is the energy sources. At present and in the foreseeable future, the viable EV energy sources are batteries, fuel cells, supercapacitors and ultrahigh-speed flywheels. Batteries are relatively the most mature source for EV application. They offer either high specific energy (HSE) or high specific power (HSP), but not both. An HSE energy source is favourable for long driving range, whereas as HSP energy source is desirable for high acceleration rate and high hill climbing capability. The electric power source for most of the two-wheelers and three-wheelers is batteries. As batteries power storage capacity is limited, these electric vehicles suffer from problem of short range. The braking performance is the basic factor of vehicle safety. In urban driving and hilly-terrain area driving, significant amount of energy is consumed in braking. The electrification of the vehicle drive train makes it feasible to recover some of the energy lost in braking by employing regenerative braking. This recovered energy can be used to increase the range of the vehicle. Depending on number of switches active during regeneration there are three types of regenerative braking methods. These are single switch braking (SSB), double switch braking (DSB) and triple switch braking (TSB).

\section{Regenerative Braking, methods and comparison}

One of the most important features of EVs, HEVs, and fuel cell vehicles (FCVs) is their ability to recover significant amounts of braking energy. The electric motors in EVs, HEVs, and FCVs can be controlled to operate as generators to convert the kinetic or potential energy of vehicle mass into electric energy that can be stored in the energy storage and then reused. The braking performance of a vehicle is an important factor in vehicle safety. A successfully designed braking system for a vehicle must always meet the distinct demand of

\footnotetext{
* Corresponding author: manish.current83@gmail.com
} 
quickly reducing vehicle speed and maintaining vehicle direction controllable by the steering wheel. The former requires the braking system to be able to supply sufficient braking torque on all wheels. Generally, the braking torque required is much larger than the torque that an electric motor can produce, especially in heavy braking. In EVs, HEVs, and FCVs, mechanical friction braking systems must coexist with electrical regenerative braking. Therefore, this is a hybrid braking system. As in the hybrid propulsion system, there are many configurations and control strategies. However, the final goal of the design and control of such systems is to ensure the vehicle's braking performance and its ability to recover as much braking energy as possible [1].

The importance of regenerative braking can be seen by knowing the fact that in typical urban areas energy required for braking operation is almost $34 \%$ of the total traction energy, while in large cities this may reach up to $80 \%$ of the total traction energy which is very high. Hence, recovering energy from the braking process can increase the range of the EV by significant distance [2].

\subsection{Regenerative Braking in Brushless DC Motor}

For an EV with brushless dc motor when the acceleration command is issued, the electric machine is operated in the motor mode (i.e. starting, acceleration, or power assisting). The output torque of the machine is controlled by adjusting the direction and amplitude of the armature current by a DC to AC inverter. In contrast, if the machine is operated in the regenerative mode (i.e. deceleration, or continuous charging), namely the deceleration command is sensed, the dynamic energy from the vehicle is transferred to the battery or capacitance via the alternator and an $\mathrm{AC}$ to $\mathrm{DC}$ converter [3].

Complete block diagram of PMBLDC motor based electric vehicle along with its control in motoring and braking region is shown in Fig: 1 . In this block diagram hybrid braking has been used. At lower speed braking torque developed by motor is not enough to stall or brake the vehicle, hence mechanical braking is preferred at lower speeds. Using this duty cycle PWM signals are generated for braking by braking decoder and PWM generator. Hence, controlled braking can be achieved by using control strategy shown in above Fig 1. If required braking torque is more than the maximum torque produced at that speed (this can be obtained by using speed- torque characteristic of motor as shown in Fig:1.) then mechanical braking must be applied. During braking mode depending on the braking torque required by driver braking current reference is generated. This reference current is compared with actual motor torque and by help of current controller duty cycle is generated.

\subsubsection{Regenerative braking with single-switch}

In this method only one power switch is operated within each commutation. The fig showing the operation with this type of switching is shown below. When braking command is given the lower switch is turned on such that the current circulates through the two switches (shown with red segments in Fig 2).In this case energy is being stored in the corresponding phase inductor. When the power switch is turned off the reversal of power takes place due to the stored energy in the inductor and hence battery can be charged during this duration (shown with dotted blue segments in Fig 2) [4]

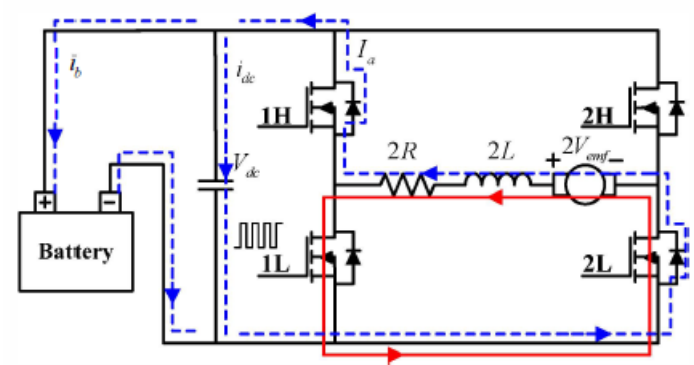

Figure 2. Circuit of the single-switch switching

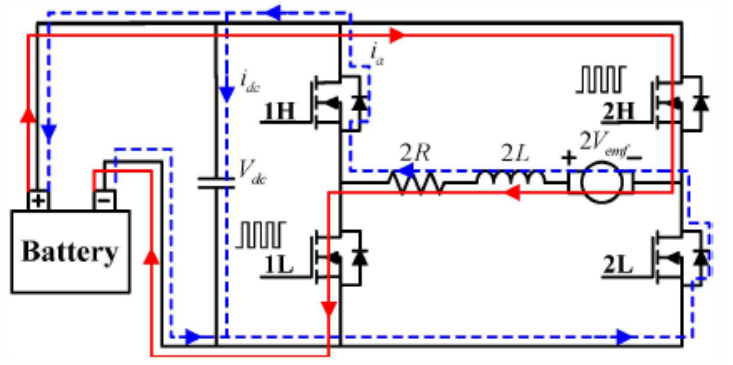

Figure 3. Circuit of the two-switches switching

\subsubsection{Regenerative braking with two-switches}

In this method two power switches are operated within each commutation. When the speed of the vehicle is relatively high, the issue of how to recover the maximum kinetic energy of the vehicles is of most importance. In contrast, for low speed operation, braking torque is the major concern. Regenerative braking with two-switches is good for low speed operation. The operation is explained with Figure 3 When braking command is given in this case the two power switches operates in such a way that, the polarity of induced voltage and the battery is additive and a large current flows through the windings of the motor, also the charging of phase inductor takes place (current path is shown in Fig 3 with red color). When switches are turned off the power reversal takes place. (shown in Figure 3 with blue dotted segment) [4-5]

\subsubsection{Regenerative braking with three-switches}

In this method three power switches are operated within each commutation. The principle of the three-switches regenerative braking is like that of the conventional dynamic braking. The current path when lower switches are turned on and off is shown in figure 4 [5]. 


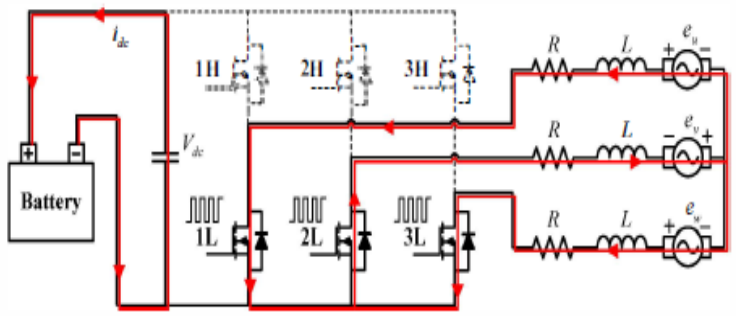

(a) Lower three switches are turned on.

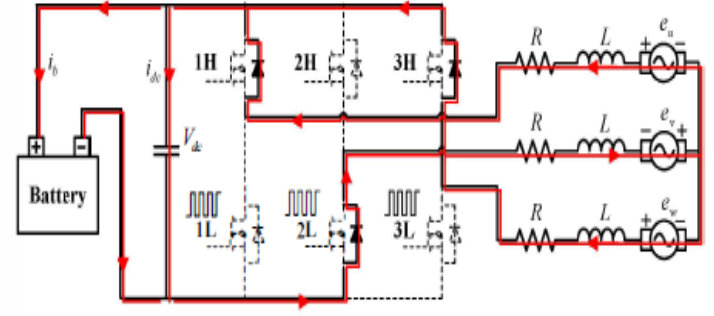

(b) Lower three switches are turned off.

Figure 4. Current paths of the three-switches switching in 30 electric degrees [5]

\subsection{Simulation Results}

Table 1. Single Switch braking with varying duty cycle and fixed speed

\begin{tabular}{|l|l|l|l|l|l|l|l|l|}
\hline S No. & Nomenclature & & \multicolumn{3}{|l|}{ Duty Cycle } \\
\hline & & 1 & 0.8 & 0.6 & 0.49 & 0.4 & 0.3 & 0.2 \\
\hline 1 & Regenerative energy (J) & 2.85 & 27.4 & 27.4 & 27 & 27 & 27 & 27 \\
\hline 2 & $\begin{array}{l}\text { Regenerative Braking } \\
\text { time (Sec) }\end{array}$ & 0.0433 & 0.085 & 0.085 & 0.167 & 0.167 & 0.167 & 0.167 \\
\hline 3 & Battery Current (A) & 60 & 37 & 37 & 14.62 & 14.5 & 14.6 & 14.5 \\
\hline 4 & Maximum Current (A) & 41 & 37 & 37 & 14.62 & 14.5 & 14.6 & 14.5 \\
\hline
\end{tabular}

Table 2. Single Switch braking with varying speed and fixed Dutycycle

\begin{tabular}{|l|l|l|l|l|l|l|l|}
\hline S No. & Nomenclature & \multicolumn{3}{|l|}{ Speed (rpm) } \\
\hline & & 300 & 275 & 250 & 225 & 200 & 175 \\
\hline 1 & Available Energy (J) & 65 & 54.61 & 45.14 & 36.56 & 28.89 & 22.11 \\
\hline 2 & Regenerative energy (J) & 27.8 & 22.71 & 18.025 & 13.47 & 9.066 & 4.51 \\
\hline 3 & Regenerative Braking time (Sec) & 0.085 & 0.077 & 0.0686 & 0.0576 & 0.0464 & 0.028 \\
\hline 4 & Battery Current (A) & 37 & 32.07 & 26.84 & 21.55 & 16.4 & 11.55 \\
\hline 5 & Maximum Phase Current (A) & 37 & 23.76 & 22.96 & 21.56 & 12 & 10.77 \\
\hline
\end{tabular}

From table 1 and 2 , it is observed that braking energy is maximum when the speed is maximum and the braking current is maximum when duty cycle is maximum. Similarly, two and three switch braking methods and the performance in each case is simulated and the results are shown in section 2.4 .

\subsection{Comparaison of regenerative braking methods}

Performance of three braking methods when duty ratio is varied and speed kept constant are compared in the following graphs 

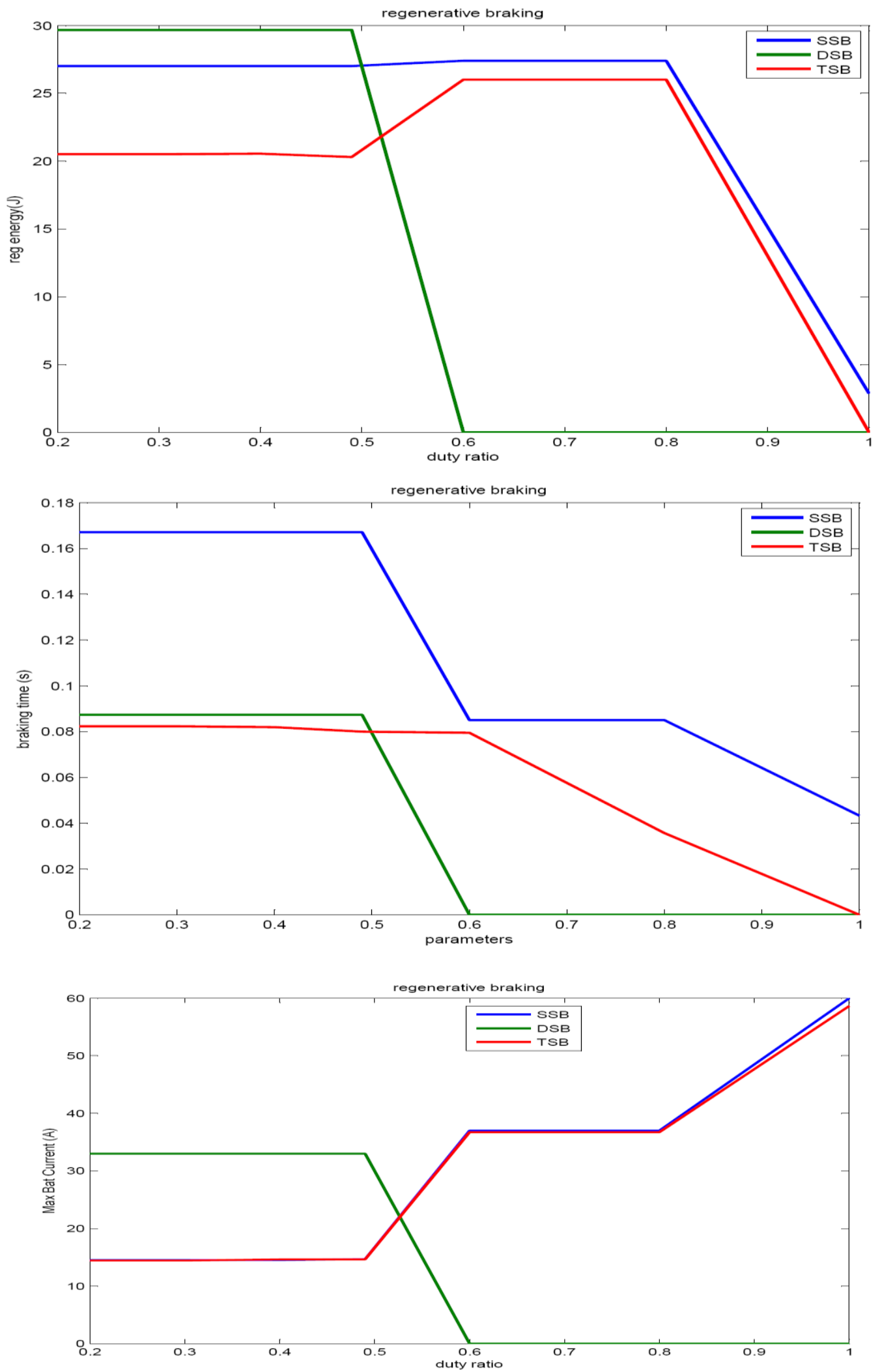

Figure 5. Comparison of three braking methods with varied duty cycle

From the above results it is observed Maximum Energy can be recovered by using double switch braking. Double switch braking method gives rise to maximum battery current during regeneration. Other two method have almost same max. battery current at low duty ratio and at higher duty ratio current in case of SSB is more than that of TSB. Braking time is least in Three Switch Braking Method. SSB method takes long braking time at low duty ratios. 


\subsection{Performance of three braking methods when speed is varied and duty ratio kept constant}
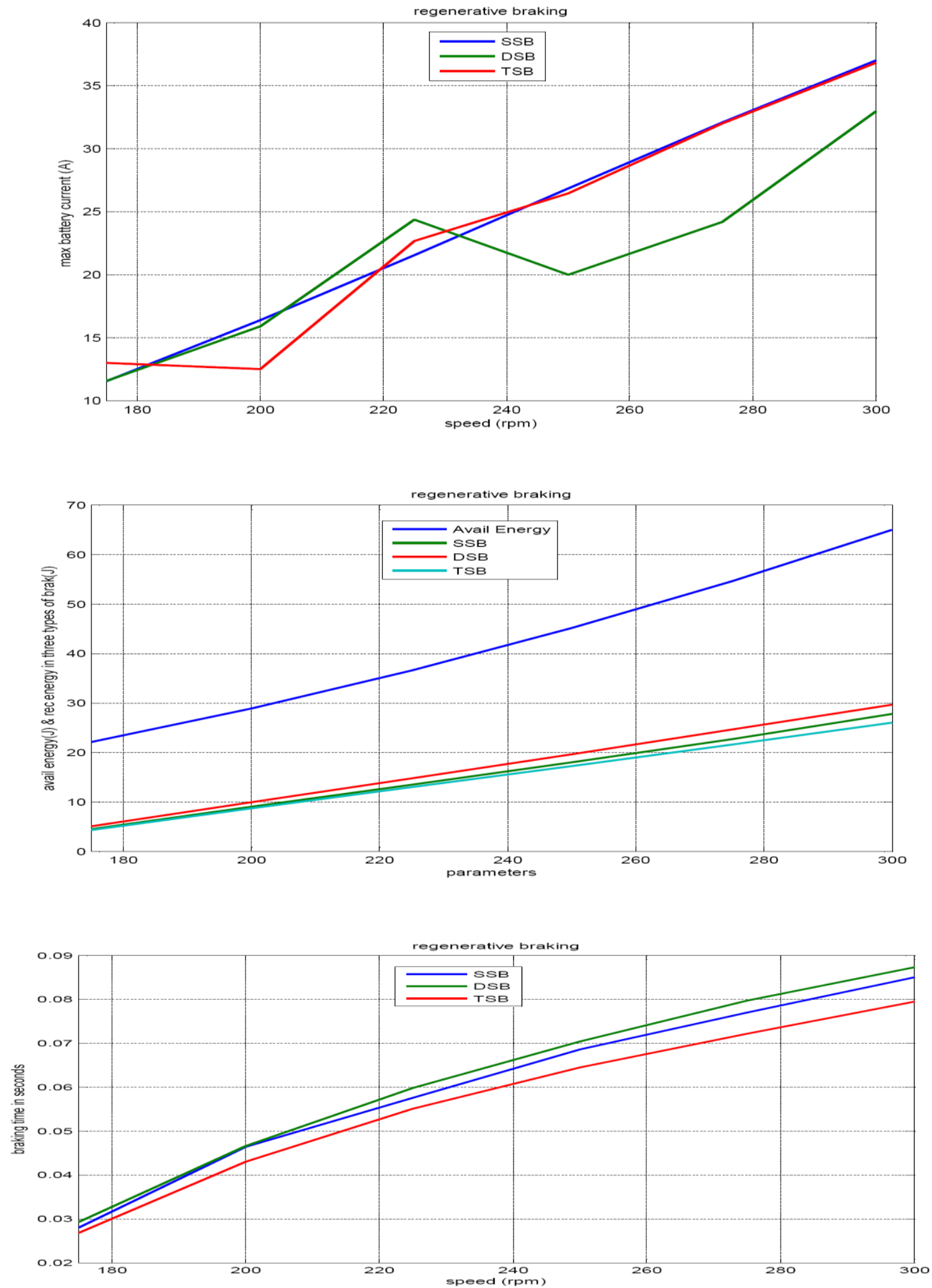

Figure 6. Comparison of three braking methods with varied Speed and Fixed Duty Cycle

From the Fig 5 and 6 it is observed that Max battery current in case of single switch braking is slightly more than that of three switch braking. and braking time is least in three switch braking method. 


\section{Conclusion}

In this paper varoious regenerative braking methods are proposed for PMBLDC Motor driven electric twowheeler. If efficiency is the major concern when the vehicle speed during the braking process is almost constant, In order to get the maximum recovered energy DSB should be used. At high speed for maximum braking torque TSB should be used, but at low speed braking torque is not sufficient in TSB, hence DSB should be used. SSB also cannot be used at low speed because of no braking torque. At high speed if energy recovery is main concern than DSB should be used.so based on the vehicle speed appropriate braking scheme is selected that means controler should works on variable switch braking scheme

\section{References}

1. C. C. Chen and K.T.Chau, Morden Elcetric Vehicle Technology, Oxford University Press, Inc., New York, 2001, page, 122-133

2. Bobba, $\mathrm{P}$ B ; Rajagopal, K R; "Compact Regenerative Braking Scheme for a PM BLDC Motor Driven Electric Two- Wheeler" Power Electronics, Drives and Energy Systems (PEDES) \& 2010 Power India, 2010 Joint International Conference.

3. Becerra, RC; Ehsani, M; Jahns,T,"Four Quadrant Brushless ECM drive with Integrated Current Regulation" Industry Applications Society Annual Meeting, 1989., Conference Record of the 1989.

4. Bobba, P B ; Rajagopal, K R; "Compact Regenerative Braking Scheme for a PM BLDC Motor Driven Electric Two- Wheeler" Power Electronics, Drives and Energy Systems (PEDES) \& 2010 Power India, 2010 Joint International Conference.

5. Cheng-Hu Chen, Wen-Chun Chi, and Ming-Yang Cheng, "Regenerative Braking Control for Light Electric Vehicles", IEEE PEDS 2011, Singapore, 5 8 December 2011

\footnotetext{
* Corresponding author: manish.current83@gmail.com
} 